\title{
International bull evaluations by genomic BLUP with a prediction population
}

\author{
B. Fragomeni, ${ }^{1,2 *}$ Y. Masuda, ${ }^{2}$ H. L. Bradford, ${ }^{3}$ D. A. L. Lourenco, ${ }^{2}$ and I. Misztal ${ }^{2}$ \\ ${ }^{1}$ Department of Animal Science, University of Connecticut, Storrs 06269 \\ ${ }^{2}$ Department of Animal and Dairy Science, University of Georgia, Athens 30602 \\ ${ }^{3}$ Department of Animal and Poultry Sciences, Virginia Tech, Blacksburg 24061
}

\section{ABSTRACT}

The purpose of this study was to determine whether multi-country genomic evaluation can be accomplished by multiple-trait genomic best linear unbiased predictor (GBLUP) without sharing genotypes of important animals. Phenotypes and genotypes with 40k SNP were simulated for 25,000 animals, each with 4 traits assuming the same genetic variance and 0.8 genetic correlations. The population was split into 4 subpopulations corresponding to 4 countries, one for each trait. Additionally, a prediction population was created from genotyped animals that were not present in the individual countries but were related to each country's population. Genomic estimated breeding values were computed for each country and subsequently converted to SNP effects. Phenotypes were reconstructed for the prediction population based on the SNP effects of a country and the prediction animals' genotypes. The prediction population was used as the basis for the international evaluation, enabling bull comparisons without sharing genotypes and only sharing SNP effects. The computations were such that SNP effects computed within-country or in the prediction population were the same. Genomic estimated breeding values were calculated by single-trait GBLUP for within-country and multiple-trait GBLUP for multi-country predictions. The true accuracy for the prediction population with reconstructed phenotypes was at most 0.02 less than the accuracy with the original data. The differences increased when countries were assumed unequally sized. However, accuracies by multiple-trait GBLUP with the prediction population were always greater than accuracies from any single within-country prediction. Multicountry genomic evaluations by multiple-trait GBLUP

Received August 14, 2018.

Accepted November 8, 2018.

*Corresponding author: breno.fragomeni@uconn.edu are possible without using original genotypes at a cost of lower accuracy compared with explicitly combining countries' data.

Key words: single nucleotide polymorphism multiple across-country evaluation, single nucleotide polymorphism effect, single-step approach, multiplecountry evaluation

\section{INTRODUCTION}

In the past, international genetic evaluations by Interbull were based on daughter yield deviations (DYD) for bulls (Schaeffer, 1994), mostly approximated as deregressed proofs. Possibly multiple DYD for each bull were used in a multiple-trait sire-model BLUP, where each country was treated as a separate trait. Pooling DYD from many countries with the multiple acrosscountry evaluation (MACE) increased reliabilities above those possible using data of any single country (Wiggans et al., 2011). Consequently, the evaluations were available for proven bulls only.

Under genomic selection, most marketed bulls are young genotyped bulls with no daughters and therefore no DYD information (Wiggans et al., 2015). Their reliability is derived via a genomic link to a reference population. The MACE could be adapted to the genomic information with genomic best linear unbiased predictor (GBLUP) if genotypes of bulls are available; however, Interbull member countries are reluctant to share genotypes. With no genotype sharing, the use of existing MACE is limited to providing external phenotypic information for national evaluations. Even such a role is under challenge when DYD are derived from BLUP as EBV from BLUP are affected by preselection (Masuda et al., 2018).

The genomic information of a reference population can be summarized in SNP effects, which, given genotypes of young animals, can be used to generate EBV for young animals. Assuming that countries are unwilling to exchange phenotypes but agree to sharing SNP effects, Goddard (2011) proposed SNP MACE. Each 
country contributes left- and right-hand sides (LHS and RHS) of SNP BLUP using the same SNP set, and all sides are put into a multiple-trait SNP BLUP. Subsequently, each country receives SNP effects on own scale while benefitting from information from other countries. Additionally, double counting of the same information in several countries can be accounted for by correlated residuals.

The use of SNP MACE has several challenges. First, different countries may be using different SNP chips, whereas SNP MACE requires identical SNP from all countries. This issue can be solved by imputation to a target chip. Second, it is not clear whether the SNP model will be computationally viable in a multiple-trait context with an almost singular variance-covariance matrix. Third, predictions need to be for SNP chips used by individual countries, although this issue can again be possibly solved by imputation.

Vandenplas et al. (2018) presented a version of SNP MACE where the submission of LHS and RHS is not required. Ideally, each country would provide SNP effects and prediction error covariances of estimated SNP effects, which have the same dimension as the LHS of SNP BLUP. When only the diagonals of prediction error covariances are available, their method becomes an approximation.

In the absence of large causative SNP, the SNP information can be seen as indirect information on independent chromosome segments (Goddard, 2009; Daetwyler et al., 2010). Subsequently, 2 genotyped subpopulations with similar reliabilities would provide almost equivalent genomic information about the chromosome segments. Thus, if submission of genotypes for a reference population is not feasible due to commercial issues, it might be possible to create an equivalent reference population with pseudo-phenotypes so that each contains identical genomic information and, when analyzed, would yield identical SNP effects to the real reference population. An equivalent reference subpopulation, which can be called the prediction population, could contain genotypes of unimportant animals from the population (e.g., cows), or artificial genotypes generated by simulating progenies of real bulls and cows. Subsequently, it may be possible to create genomic MACE based on GBLUP. Each country would submit genotypes and pseudo-phenotypes of an equivalent subpopulation. Subpopulations from multiple countries would be analyzed by multiple-trait GBLUP that works well in multiple-trait scenarios. Predictions on each country scale would be converted to any desired set of SNP effect (VanRaden, 2008; Stranden and Garrick, 2009). Inverses of the large-scale genomic relationship matrix could be computed by the algorithm for proven and young animals (Fragomeni et al., 2015; Misztal,
2016) efficiently for any number of genotyped animals, eliminating computing restrictions.

The use of equivalent subpopulations would eliminate the need to share genotypes of important animals. As GBLUP MACE is based on many assumptions, the purpose of this study is to evaluate GBLUP MACE by simulation.

\section{MATERIALS AND METHODS}

Animal Care and Use Committee approval was not obtained for this study because no animals were used.

\section{Simulation}

Using the software QMSim (Sargolzaei and Schenkel, 2009), we simulated a livestock population under selection for a single quantitative trait that has a heritability of 0.25 . A total of 5 replicates of QMSim were generated. A historical population started from 1,000 founders and increased to 100,000 after 1,000 generations, with equal proportion of males and females. This step created initial linkage disequilibrium, mutation, and drift. A total of 30 males and 20,000 females were selected from the last generation of historical population to create a recent population with effective size $(\mathrm{Ne})$ of 120. After 20 overlapping generations of selection, the recent population consisted of 487,909 animals. Full sib groups of 10 ( $1 \%$ of females), $5(2 \%)$, and 1 (97\%), were simulated to represent embryo transfer. Male to female ratio was 1:1. Sire and dam replacement rates were 90 and $30 \%$, respectively, and mating was random. Only genotyped animals were used for analyses.

Genomic information was available only for animals in the last 5 generations. Twenty thousand animals with progeny were genotyped in generations 15 to 19 , and $5 \mathrm{k}$ animals were randomly genotyped in generation 20 . We simulated 29 chromosomes mimicking the bovine genome, with 45,000 SNP evenly spaced across all the genome. Each chromosome contained between 9 and 34 biallelic randomly located QTL (casual variants), this value was proportional to each chromosome's length, totaling 500 QTL across the whole simulated genome. The QTL effects were sampled from a gamma distribution with a shape parameter of 0.4 , scaled internally for a genetic variance of 0.25 , and explained $100 \%$ of the genetic variance of the trait.

To simulate international evaluations, multiple traits were simulated, where each trait represented a different country. The single trait breeding values from QMSim were used to create breeding values for 3 more correlated traits representing 4 total countries. These breeding values $(\mathbf{u})$ were generated for the $25 \mathrm{k}$ genotyped animals as follows: 


$$
\mathbf{u} \sim \operatorname{MVN}\left(0, \mathbf{G}_{0} \otimes \mathbf{G R M}\right),
$$

where $\mathbf{G}_{0}$ is a variance-covariance matrix among countries assuming genetic variance of 0.25 and genetic correlation of 0.8 :

$$
\mathbf{G}_{0}=\left[\begin{array}{cccc}
0.25 & 0.20 & 0.20 & 0.20 \\
0.20 & 0.25 & 0.20 & 0.20 \\
0.20 & 0.20 & 0.25 & 0.20 \\
0.20 & 0.20 & 0.20 & 0.25
\end{array}\right],
$$

and GRM is a genomic relationship matrix for the 25k animals based on 500 causative QTL. GRM was calculated following Fragomeni et al. (2017):

$$
\mathbf{G R M}=\mathbf{Z}_{q} \mathbf{D} \mathbf{Z}_{q}^{\prime} / \sum_{i=1}^{q} 2 \mathbf{p}_{i}\left(1-\mathbf{p}_{i}\right),
$$

where $\mathbf{Z}_{q}$ is the incidence matrix with 0,1 , and 2 for the causative QTL; $\mathbf{p}_{i}$ is the minor allele frequency of the $i$ th causative QTL; and $\mathbf{D}$ is a diagonal matrix with elements equal to square of simulated causative QTL effect.

Because DYD (or their equivalents such as deregressed proofs) were typically shared in international dairy evaluations, the simulated phenotypes were assumed to be DYD. These DYD were assumed to have the same reliability within each country, and 0.83, 0.71, and 0.63 reliabilities were simulated for 3 scenarios. These DYD were calculated as breeding values plus a random noise distributed as $N\left(\mathbf{0}, \sigma_{e}^{2}\right)$, with $\sigma_{e}^{2}$ equal to $0.05,0.1$, and 0.15 , generating decreasing reliabilities. Within each QMSim replicate, the DYD were replicated 10 times.

\section{Multi-Country Scheme and Prediction Population}

The 20k progeny-tested, genotyped animals were split into 4 different countries. Several scenarios were considered:

1. 5k animals were common to all countries. Reference population consisted of $5 \mathrm{k}$ animals with $100 \%$ overlapping among countries.

2. $2.5 \mathrm{k}$ animals were common to all countries. Additional $2.5 \mathrm{k}$ animals were unique to each country. Therefore, all the countries had $50 \%$ of animals in common. Reference population for each country consisted of $5 \mathrm{k}$ animals, and a total of $10 \mathrm{k}$ animals were present in the reference population.
3. $5 \mathrm{k}$ animals were unique to each country with no animals shared between countries, totaling $20 \mathrm{k}$ animals in all 4 populations.

4. An additional scenario was tested with variable numbers of animals per country and no overlap of animals among countries (i.e., $1 \mathrm{k}, 2 \mathrm{k}, 4 \mathrm{k}$, or $5 \mathrm{k})$.

The prediction population contained $5 \mathrm{k}$ young animals and was used for validation. This population did not belong to any country but was related to animals in all countries. Prediction animals had genotypes but no phenotypes. Within-country DYD were reconstructed based on SNP effects from that country as detailed in the next section. Hence, prediction animals had reconstructed DYD $\left(\mathbf{D Y D}_{\mathbf{r}}\right)$ for each country resulting in 4 $\mathrm{DYD}_{\mathrm{r}}$ per prediction animal. These $\mathrm{DYD}_{\mathrm{r}}$ were used in analyses specifying reconstructed phenotypes, otherwise the prediction population had no phenotypes, much like traditional validations.

\section{Calculating Genomic EBV, SNP Effects, and DYDr}

Within-country genomic EBV (GEBV) were computed by a single-trait GBLUP:

$$
\left(\mathbf{I}+\mathbf{G R M}^{-1} \beta\right) \hat{\mathbf{u}}=\mathbf{y}
$$

where $\hat{\mathbf{u}}$ is GEBV, $\mathbf{y}$ is the vector with DYD, $\mathbf{I}$ is the identity matrix, $\mathbf{G R M}^{-1}$ is the inverse of the genomic relationship matrix, and $\beta=\sigma_{e}^{2} / 0.25$ is the variance ratio.

The SNP effects for each country were calculated from GEBV (VanRaden, 2008; Stranden and Garrick, 2009) as

$$
\hat{\mathbf{a}}=\lambda \mathbf{Z}^{\prime} \mathbf{G R M}^{-1} \hat{\mathbf{u}},
$$

where $\mathbf{a}$ is the vector containing estimated SNP effects, $\lambda=1 / \sum_{i=1}^{m} 2 p_{i}\left(1-p_{i}\right)$, where $p_{i}$ is the allelic frequency of the ith marker, and $\mathbf{Z}$ is the SNP content matrix; the dimension of $\mathbf{Z}$ is the number of animals by the number of SNP markers. Once SNP effects are calculated, genomic predictions can be obtained for young genotyped animals by multiplying the SNP content by SNP effect. GEBV contains a fraction of the polygenic effect if the genomic relationship matrix is blended with a portion of the pedigree relationship matrix. This is usually done in GBLUP to avoid singularity problems.

The $\mathrm{DYD}_{\mathrm{r}}$ for each specific country were calculated from within-country SNP effects, assuming all 4 coun- 
tries and the prediction population shared the same SNP:

$$
\mathbf{y}^{*}=\left(\mathbf{I}+\mathbf{G R M}^{-1} \beta\right) \mathbf{Z} \hat{\mathbf{a}}
$$

where $\mathbf{y}^{*}$ was the vector containing the $\mathrm{DYD}_{\mathrm{r}}$.

\section{Analyses}

To evaluate the accuracy of prediction using $\mathrm{DYD}_{\mathrm{r}}$ as pseudo-phenotype, the following methods were compared:

- Single-trait GBLUP (ST-GBLUP): withincountry model with DYD and genotypes.

- Single-trait SNP-BLUP: similar to ST-GBLUP, but using SNP-BLUP instead.

- Multiple-trait GBLUP: multi-country model with each country as a different trait and all countries providing DYD and genotypes.

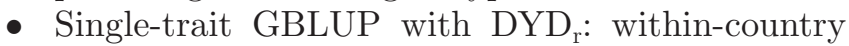
model with only $\mathrm{DYD}_{\mathrm{r}}$ and genotypes for the prediction population.

- Multiple-trait GBLUP with $\mathrm{DYD}_{\mathrm{r}}$ : multi-country model with each country as a different trait and using only $\mathrm{DYD}_{\mathrm{r}}$ and genotypes for the prediction population.

Methods were compared by true accuracies calculated as the correlation between the estimated and simulated breeding values for prediction animals.

\section{Algorithm}

The analysis proceeded as follows:

1. For each reference population (i.e., each different country):

a. Calculate GEBV using GBLUP;

b. Convert GEBV to SNP effects.

2. For the prediction population calculate $\mathrm{DYD}_{\mathrm{r}}$ using SNP effects from the reference population.

3. Treating $\mathrm{DYD}_{\mathrm{r}}$ from each country as a different trait, calculate GEBV for each country by multiple-trait GBLUP.

4. If SNP effects by country are desired, convert GEBV from each country to SNP effects.

\section{RESULTS AND DISCUSSION}

As a check on data simulation, variance components were estimated for the data sets using REML (Table 1). Variances were slightly overestimated, with the genetic correlations as simulated. The overestimation could be
Table 1. Variance components of 4-trait simulation, averaged over 10 replicates $^{1}$

\begin{tabular}{lcccc}
\hline Country & 1 & 2 & 3 & 4 \\
\hline 1 & $\mathbf{0 . 2 7}^{2}$ & 0.21 & 0.21 & 0.21 \\
2 & 0.80 & $\mathbf{0 . 2 7}$ & 0.22 & 0.21 \\
3 & 0.80 & 0.80 & $\mathbf{0 . 2 7}$ & 0.27 \\
4 & 0.79 & 0.80 & 0.78 & $\mathbf{0 . 2 7}$ \\
\hline
\end{tabular}

${ }^{1}$ Genetic (co)variances on diagonal and above diagonal and genetic correlations below diagonal.

${ }^{2}$ Standard error for all estimates was $<0.01$.

due to using only causative SNP in the construction of the genomic relationship matrix during the data simulation.

Standard error of accuracies between the 5 QMSim runs was below 0.01; therefore, only results for the first run are shown. Accuracies reported below are averages of 10 replicates for DYD generation. Values from STGBLUP and SNP-GBLUP were the same; therefore, only the first one is reported.

Table 2 presents average across-country accuracies for countries of equal size $(5 \mathrm{k})$, different level of overlap, and reliabilities of DYD; accuracies presented were averaged across countries. Standard errors of accuracies when using 10 replicates for the DYD, and across the 4 countries, were lower than 0.01 . In all cases, using the information across countries (multiple-trait) increased accuracies by up to 0.04 . Use of prediction populations with reconstructed phenotypes reduced the accuracies at most by 0.02 in either within-country (single-trait) or multi-country analyses. The percent of genotyped animals in common among countries did not change the results much, suggesting that the use of the prediction population is sound for populations with any number of genotypes in common. However, the total number of genotyped animals should be increased to counterbalance a smaller overlap. The reference population size will depend on population structure and dimensionality of genomic information (Misztal, 2016). Different reliabilities for 100 and $50 \%$ overlapping showed the same trends as for $0 \%$ overlapping (results not shown).

With greater reliabilities, the accuracies increased and differences between within- and multi-country analyses decreased. The opposite was observed with lesser DYD reliabilities. Regarding the comparison of multi-country with original data or prediction populations' data, using the latter slightly reduced accuracies. When using multiple countries with the prediction population, accuracies were always greater than withincountry accuracies without the prediction population.

Table 3 presents accuracies obtained for populations of unequal size: $5 \mathrm{k}, 4 \mathrm{k}, 2 \mathrm{k}$, and $1 \mathrm{k}$. For individual countries, gains of accuracies using multiple-trait GBLUP were greater for smaller countries, reaching 0.21 for 
Table 2. Accuracies $(\times 100)$ for 4 different models when daughter yield deviations (DYD) have different reliabilities and countries have 0 , 50 , or $100 \%$ of genotyped animals in common ${ }^{1}$

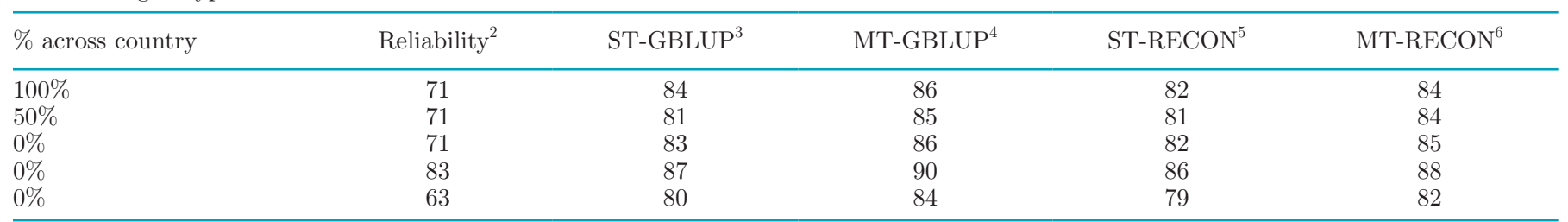

${ }^{1}$ All SE $<0.01$.

${ }^{2}$ Average reliability of DYD.

${ }^{3}$ Single-trait analysis using DYD. GBLUP = genomic best linear unbiased predictor.

${ }^{4}$ Multi-trait analysis using DYD.

${ }^{5}$ Single-trait analysis using reconstructed DYD.

${ }^{6}$ Multi-trait analysis using reconstructed DYD.

the country with $1 \mathrm{k}$ animals. This is understandable as smaller populations benefit more from information in larger populations than vice versa. For prediction populations, within-country accuracies when using $\mathrm{DYD}_{\mathrm{r}}$ were at most 0.01 less than without reconstructed phenotypes. For multi-country analyses, the accuracy improvement with $\mathrm{DYD}_{\mathrm{r}}$ was less, and for the $1 \mathrm{k}$ country the improvement was only 0.07 instead of 0.21 . It is not clear whether lower accuracies with variable-sized countries are real or artifacts of data simulation.

In this study, we use a single prediction population for all the countries. In such a scheme, an international organization (such as Interbull) would provide genotypes of animals, and individual countries would assign those animals pseudo-observations $\left(\mathrm{DYD}_{\mathrm{r}}\right)$ based on SNP effects in that country and reliabilities of their reference population. Those genotypes could be any permissible subset of genotyped animals, such as animals not important commercially, simulated mating of genotyped animals, or simulated to resemble the target population. Use of one prediction population simplifies computations and quality control but could be restrictive (e.g., when the size is insufficient). Otherwise, each country would create and submit its own prediction population.
Whether GBLUP MACE is of value requires further studies. On the one hand, it seems that GBLUP MACE may have some merit as accuracies with multi-country prediction populations were always greater than accuracies for any single country. Also, computations are minimal and can be done for a large number of countries and animals. On the other hand, several problems remain, including creation of within-country reference populations and reasons for reduced accuracies in countries with unequal size. In this study, the countries were composed of animals with equal reliability, and the prediction animals had the same characteristics. Real reference populations have a complex structure with animals of variable reliability. An important question is how to create a simple prediction population that has the same genomic information as multiple countries' populations. Our prediction population had strong relationships with each country because the last simulated generation was used for prediction. Further research is needed in this area. Another question is whether GBLUP MACE would retain good accuracies in complex data structures. Assuming same traits in all populations, with SNP MACE (Goddard, 2011) all LHS and RHS are added, and the results are the same as with a combined analysis. The same will be true

Table 3. Accuracies $(\times 100)$ for 4 countries with unequal size and by 4 methods ${ }^{1}$

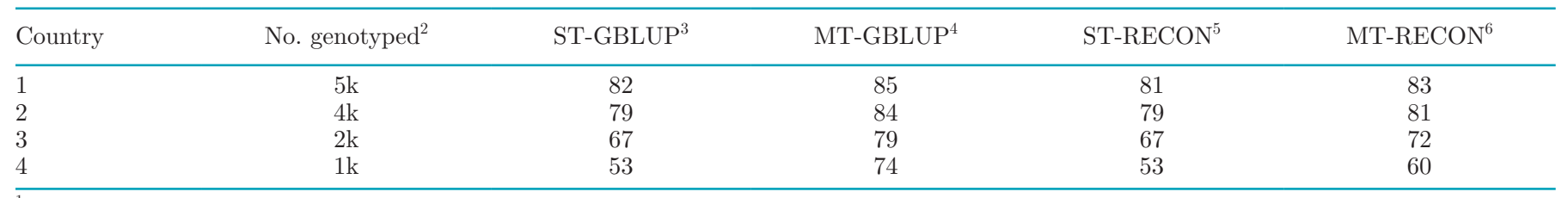

${ }^{1}$ Reliability of daughter yield deviations (DYD) was 71 .

${ }^{2}$ Number of genotyped animals in each country, where $\mathrm{k}=1,000$ individuals.

${ }^{3}$ Single-trait analysis using DYD. GBLUP = genomic best linear unbiased predictor.

${ }^{4}$ Multi-trait analysis using DYD.

${ }^{5}$ Single-trait analysis using reconstructed DYD.

${ }^{6}$ Multi-trait analysis using reconstructed DYD. 
with GBLUP and original data sets, but not necessarily with prediction populations.

Both SNP MACE and GBLUP MACE face the issue of quality of DYD. Under genomic selection, BLUP is biased by preselection, DYD directly obtained from BLUP are biased (Patry and Ducrocq, 2011; Masuda et al., 2018), and heuristics are used to remove this bias (Mäntysaari and Strandén, 2010; VanRaden, 2012; Stoop et al., 2013). As heuristics are likely to become more complex across countries, probably national DYD would have to be derived by a method that explicitly accounts for the bias [e.g., single-step whether based on GBLUP (Aguilar et al., 2010) or Bayesian regressions (SSBR; Fernando et al., 2016)].

The GBLUP MACE shares the issue of SNP MACE that it allows to backsolve for SNP effects, which are treated by many countries as proprietary information. Several choices may be applicable to secure countries' interests. First, for sparsely recorded traits where no country has sufficient phenotypes for reliable predictions, pooling may be beneficial for all the countries. Second, the largest countries may release only a fraction of their genomic information, incentivizing data sharing. Finally, when the size of the reference populations in many countries becomes very large, SNP effects may functionally converge and become commodities, making genomic MACE more of a quality control and country-scaling tool than an industry secret.

\section{CONCLUSIONS}

The use of GBLUP MACE allows for multi-country genomic evaluation where countries are not required to submit original genotypes or phenotypes. In comparisons, the method produced lower accuracy than with explicitly combined populations but higher accuracy than with any single country. The MACE GBLUP is easy to apply as the exiting MACE software can be modified by replacing the inverse of the numerator relationship matrix by the inverse of the genomic relationship matrix, possibly using the algorithm for proven and young animals to eliminate restrictions on the number of genotyped animals.

\section{ACKNOWLEDGMENTS}

Helpful comments by Hossein Jorjani (Interbull Centre, Uppsala, Sweden), Tom J. Lawlor (Holstein Association USA, Brattleboro, VT), and Paul M. VanRaden (Animal Genomics and Improvement Laboratory, USDA, Beltsville, MD) are gratefully acknowledged. This research was primarily supported by grants from Holstein Association USA and the USDA National
Institute of Food and Agriculture (Washington, DC; Agriculture and Food Research Initiative competitive grant 2015-67015-22936).

\section{REFERENCES}

Aguilar, I., I. Misztal, D. L. Johnson, A. Legarra, S. Tsuruta, and T. J. Lawlor. 2010. Hot topic: a unified approach to utilize phenotypic, full pedigree, and genomic information for genetic evaluation of Holstein final score. J. Dairy Sci. 93:743-752.

Daetwyler, H. D., R. Pong-Wong, B. Villanueva, and J. A. Woolliams. 2010. The impact of genetic architecture on genome-wide evaluation methods. Genetics 185:1021-1031.

Fernando, R. L., H. Cheng, B. L. Golden, and D. J. Garrick. 2016. Computational strategies for alternative single-step Bayesian regression models with large numbers of genotyped and non-genotyped animals. Genet. Sel. Evol. 48:96.

Fragomeni, B. O., D. A. Lourenco, S. Tsuruta, Y. Masuda, I. Aguilar, A. Legarra, T. J. Lawlor, and I. Misztal. 2015. Hot topic: Use of genomic recursions in single-step genomic best linear unbiased predictor (BLUP) with a large number of genotypes. J. Dairy Sci. 98:4090-4094.

Fragomeni, B. O., D. A. L. Lourenco, Y. Masuda, A. Legarra, and I. Misztal. 2017. Incorporation of causative quantitative trait nucleotides in single-step GBLUP. Genet. Sel. Evol. 49:59.

Goddard, M. 2009. Genomic selection: Prediction of accuracy and maximisation of long term response. Genetica 136:245-257.

Goddard, M. 2011. An international SNP model. Paper presented at the Interbull Technical Workshop, Guelph, Canada.

Mäntysaari, E. A., and I. Strandén. 2010, Use of bivariate EBV-DGV model to combine genomic and conventional breeding value evaluations. Paper presented at the 9th World Congress on Genetics Applied to Livestock Production, Leipzig, Germany, Aug. 1-6.

Masuda, Y., P. M. VanRaden, I. Misztal, and T. J. Lawlor. 2018. Differing genetic trend estimates from traditional and genomic evaluations of genotyped animals as evidence of preselection bias in US Holsteins. J. Dairy Sci. 101:5194-5206.

Misztal, I. 2016. Inexpensive computation of the inverse of the genomic relationship matrix in populations with small effective population size. Genetics 202:401-409.

Patry, C., and V. Ducrocq. 2011. Accounting for genomic pre-selection in national BLUP evaluations in dairy cattle. Genet. Sel. Evol. 43:30.

Sargolzaei, M., and F. S. Schenkel. 2009. QMSim: A large-scale genome simulator for livestock. Bioinformatics 25:680-681.

Schaeffer, L. R. 1994. Multiple-country comparison of dairy sires. J. Dairy Sci. 77:2671-2678.

Stoop, W. M., H. Eding, M. L. van Pelt, L. C. M. de Haer, and G. de Jong. 2013. Using pseudo-observations to combine genomic and conventional data in the Dutch-Flemish national evaluation. Interbull Bull. 47:106-110.

Stranden, I., and D. J. Garrick. 2009. Technical note: Derivation of equivalent computing algorithms for genomic predictions and reliabilities of animal merit. J. Dairy Sci. 92:2971-2975.

Vandenplas, J., M. P. L. Calus, and G. Gorjanc. 2018. Genomic prediction using individual-level data and summary statistics from multiple populations. Genetics https://doi.org/10.1534/genetics .118 .301109 .

VanRaden, P. M. 2008. Efficient methods to compute genomic predictions. J. Dairy Sci. 91:4414-4423.

VanRaden, P. M. 2012. Avoiding bias from genomic pre-selection in converting daughter information across countries. Interbull Bull. 45:29-33.

Wiggans, G. R., P. M. Vanraden, and T. A. Cooper. 2011. The genomic evaluation system in the United States: Past, present, future. J. Dairy Sci. 94:3202-3211.

Wiggans, G. R., P. M. VanRaden, and T. A. Cooper. 2015. Technical note: Rapid calculation of genomic evaluations for new animals. J. Dairy Sci. 98:2039-2042. 